\title{
Case Report \\ Scoliosis Surgery in Cystic Fibrosis: Surgical Considerations and the Multidisciplinary Approach of a Rare Case
}

\author{
George I. Mataliotakis, Athanasios I. Tsirikos, Karen Pearson, Don S. Urquhart, \\ Carolyn Smith, and Andrew Fall
}

Scottish National Spine Deformity Centre, Royal Hospital for Sick Children, Sciennes Road, Edinburgh EH9 1LF, UK

Correspondence should be addressed to Athanasios I. Tsirikos; atsirikos@hotmail.com

Received 9 April 2016; Accepted 30 May 2016

Academic Editor: Hitesh N. Modi

Copyright (c) 2016 George I. Mataliotakis et al. This is an open access article distributed under the Creative Commons Attribution License, which permits unrestricted use, distribution, and reproduction in any medium, provided the original work is properly cited.

\begin{abstract}
Spinal deformity in patients with cystic fibrosis $(\mathrm{CF})$ is usually mild requiring no treatment. These patients are rarely considered as surgical candidates for scoliosis correction, as the pulmonary condition and other comorbidities increase the risk of general anaesthesia and recovery. This paper reviews all the literature up to date with regard to scoliosis in patients with CF and reports this unique case of a 14-year-old Caucasian girl with progressive scoliosis, who was treated surgically at the age of 17 . She underwent a posterior spinal fusion T2-L3 with the use of unilateral segmental instrumentation. Preoperative workup included respiratory, cardiac, anaesthetic, endocrine, and dietician reviews, as well as bone density optimisation with zoledronic acid and prophylactic antibiotics. Surgical time was 150 minutes and intraoperative blood loss was $47 \%$ of total blood volume. Postoperative intensive care included noninvasive ventilation, antibiotic cover, pain management, chest physiotherapy, pancreatic enzyme supplementation, and nutritional support. She was discharged on day 9. At follow-up she had a good cosmetic outcome, no complaints of her back, and stable respiratory function. Multidisciplinary perioperative care and meticulous surgical technique may reduce the associated risks of major surgery in CF patients, while achieving adequate deformity correction and a good functional outcome.
\end{abstract}

\section{Introduction}

Cystic fibrosis (CF) is the most common life-limiting autosomal recessive disease in the United Kingdom, affecting over 10,000 children and young adults. Average life expectancy is currently 41 years [1]. Chronic mucus hypersecretion leads to recurrent lower respiratory tract infections and lung disease, while nutrition and bone health may be adversely impacted upon due to exocrine pancreatic insufficiency, vitamin deficiencies, and inflammation.

A number of later-onset musculoskeletal manifestations of $\mathrm{CF}$ are reported including scoliosis, kyphosis, low bone mineral density, and pathological fractures [2-10].

Case series of children and adolescents with CF and scoliosis have been reported by a number of centres including our own centre $[2-4,7,9,10]$. However, the paradigm of a radiologically significant (Cobb angle $>10^{\circ}$ ) versus a clinically important scoliosis remains a key message from these reports. Reduced respiratory reserve when present due to underlying lung disease may make CF patients unsuitable surgical candidates; however the spinal deformity is usually mild and does not require surgical correction $[3,7,10]$.

We report a young woman with $\mathrm{CF}$ and progressive thoracic scoliosis, who underwent surgical treatment. To our knowledge, this is the first report of a CF patient who underwent posterior spinal fusion for scoliosis.

\section{Case Report}

2.1. History and Examination. A Caucasian girl with $\mathrm{CF}$ (genotype: F508del/F508del) was referred to clinic with a double thoracic scoliosis, at the age of 14.3 years. This was an incidental finding when a chest X-ray was obtained as part of regular respiratory reviews. On examination, she had a rib hump and small thoracic translocation to the right with associated waistline asymmetry and prominence of the left side of the pelvis. Her shoulders and pelvis were level. 
Neurological examination and spinal MRI were normal. Mild ligamentous laxity was detected. Spinal radiographs showed a left upper thoracic and right low thoracic curve measuring $23^{\circ}$ and $36^{\circ}$, respectively. Sagittal balance was normal and the Risser grade was 0 . She was 1.3 years after menarche with positive history of mild scoliosis in the maternal extended family, which never required treatment.

2.2. Treatment. An underarm brace was attempted with the aim to stop scoliosis progression but was poorly tolerated by the patient. Over 2.5 years of monitoring both curves gradually deteriorated and the patient developed persistent mechanical back pain. Scoliosis correction was offered and a detailed preoperative assessment (cardiac, respiratory, anaesthetic, endocrine, and dietician) was organised. The cardiac review showed no anomalies.

2.3. Respiratory. Lung function had been stable for many months prior to surgery (FEV-1: 1.66 litres/59\% predicted; FVC: 2.25 litres/69\% predicted) and oxygen saturation was $98 \%$ in air; no further lung plethysmography was performed. Her sputum was chronically infected with Stenotrophomonas maltophilia and Methicillin-Resistant Staphylococcus aureus. Pseudomonas aeruginosa had been previously isolated and she had also had treatment for allergic bronchopulmonary Aspergillosis (ABPA). She had undergone Nissen fundoplication aged 6 years due to severe gastroesophageal reflux. She had severe bilateral bronchiectasis demonstrable on CT scan and received regular courses of intravenous antibiotics, daily nebulised mucolytic (dornase-alfa) and antibiotic (alternating colistin/tobramycin) treatments. She had a portacath (Port-a-Cath ${ }^{\mathrm{TM}}$ ) implantable venous access device. In addition, she received nutritional supplements, pancreatic enzyme replacement therapy, and daily supplementation of calcium, as well as vitamins A, D, E, and K. Her body weight was $54 \mathrm{~kg}$ with normal BMI. She has had previous adverse reactions to antibiotics (vancomycin, ceftazidime, teicoplanin, piperacillin/tazobactam, ciprofloxacin, and levofloxacin) and received intravenous (IV) aztreonam and cotrimoxazole for 2 weeks before surgery.

2.4. Endocrine. Bone mineral density (BMD-lumbar spine DEXA scan) $Z$ scores had been consistently $<-2.5$ since the age of 12. Zoledronic acid was administered IV 4 months prior to surgery in addition to her long-term calcium and vitamin D3 supplementation to improve bone strength.

2.5. Scoliosis Surgery. She underwent a posterior spinal fusion T2-L3 with the use of a single pedicle hook/screw/rod construct. Due to increased bleeding the side of the instrumentation was exposed first and the construct was finalised before the contralateral side was grafted with the use of autologous and allograft bone. Surgical time (skin incision to closure) was 150 minutes and blood loss $1700 \mathrm{~mL}$ (47\% of total blood volume). The patient received transfusion of one unit of packed red cells, $260 \mathrm{~mL}$ of cell-salvaged blood, and 3 units of fresh frozen plasma (FFP) along with $1500 \mathrm{~mL}$ crystalloid fluid. Spinal cord monitoring (motor/sensory evoked potentials, EMG) was stable throughout the procedure.

2.6. Anaesthetic. Previous anaesthetics included laparoscopic fundoplication, portacath insertion, dental extractions, and numerous bronchoscopies. The portacath was utilised for induction of anaesthesia with initial propofol bolus of $120 \mathrm{mg}$ followed by initiation of maintenance with total intravenous anaesthesia propofol and remifentanil target-controlled infusions and paralysis with atracurium. Her airway was easily managed and a size $6.5 \mathrm{~mm}$ reinforced endotracheal tube was sited with laryngoscopy direct grade 1 view. Several extra large-bore IV cannulae, a left radial arterial line, and a urinary catheter were then sited along with BIS forehead sensors for depth of anaesthesia monitoring. Active warming measures were employed and normothermia was achieved during procedure. Intraoperative analgesia included morphine, clonidine, and paracetamol. Initial tranexamic acid bolus of 2 grams was followed by an infusion of $500 \mathrm{mg} /$ hour. Arterial systolic blood pressure was maintained between 85 and $100 \mathrm{mmHg}$ throughout surgery with titration of GTN and remifentanil. The patient was successfully extubated at the end of the procedure, remained haemodynamically stable in recovery, and was transferred to PICU for ongoing care.

2.7. Postoperative Care. The patient was placed on noninvasive positive pressure ventilation. The pressures were weaned on day 2 postoperatively and the patient managed time off NIV on days 2 and 3, switching to nocturnal-only NIV support on day 3 with spontaneous ventilation on room air thereafter. She remained haemodynamically stable throughout her 4-day admission to PICU, alert, and interacting well with staff. She received a blood transfusion on day 3 in response to haemoglobin of $70 \mathrm{~g} / \mathrm{dL}$. Beclomethasone and salbutamol inhalers were restarted; antibiotic cover included aztreonam, cotrimoxazole, linezolid, and fluconazole as per microbiology recommendations. Pain management included IV morphine supplemented by clonidine, diazepam, paracetamol, and ibuprofen. This was stepped down to fentanyl patch and breakthrough sevredol on day 3. Pancreatic enzymes and vitamins were given as preoperatively along with supportive medication (ondansetron, cyclizine, omeprazole, macrogol, and picosulfate laxatives). She was transferred to the respiratory ward on day 4 after surgery.

Intensive chest physiotherapy and early mobilisation gradually improved right basal consolidation and the antibiotics were stopped on day 7 after surgery. Postoperative $\mathrm{X}$-rays showed excellent correction of the double thoracic scoliosis and a balanced spine in both planes (Figure 1). She was fitted with a removable custom-moulded spinal jacket to wear when out of bed for 6 months in order to support the spine as the bone grafts were healing. She was discharged on the 9th postoperative day on paracetamol, tramadol, and gabapentin. Supplements were given beyond discharge until oral intake was optimised. At follow-up she had a well-healed scar, good spinal balance in the frontal and lateral planes, normal chest X-ray, and no respiratory or spinal complaints. 


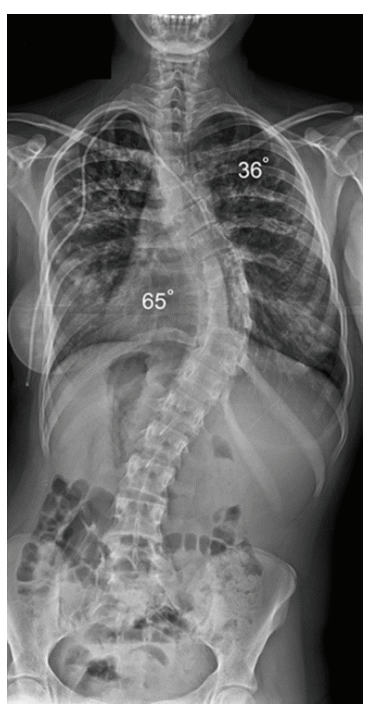

(a)

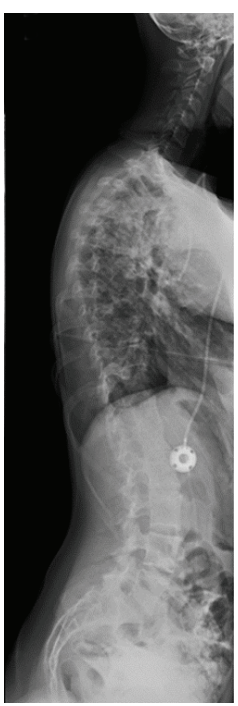

(b)

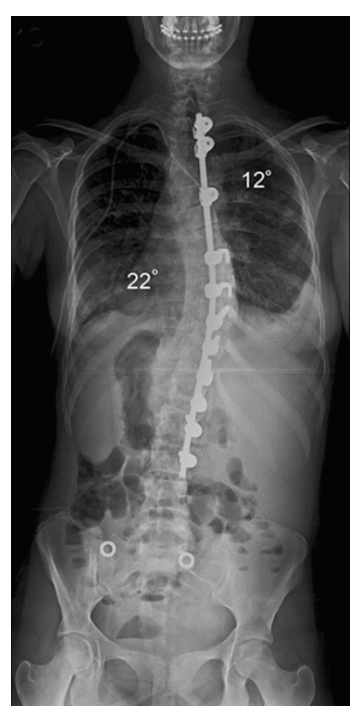

(c)

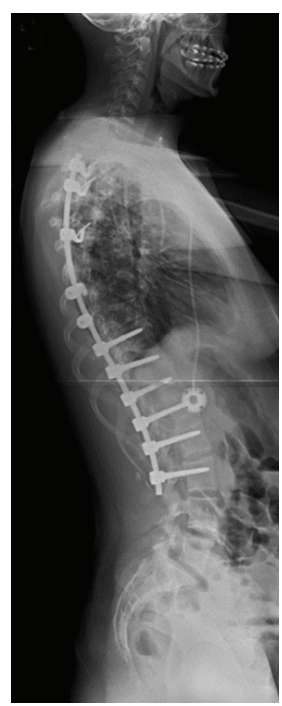

(d)

FIGURE 1: Preoperative posterior-anterior (a) and lateral (b) radiographs of the spine show a left upper and right low thoracic scoliosis measuring 36 and $65^{\circ}$, respectively. Posterior spinal fusion with the use of a right hook/screw and rod construct has achieved excellent correction of the curves to 12 and $22^{\circ}$ and a satisfactory global coronal and sagittal balance for the spine (c, d).

\section{Discussion}

The prevalence of scoliosis in CF ranges from 9.9 to $15.5 \%$, has features of adolescent idiopathic scoliosis, and is greater in children older than 10 years (Table 1$)[3,7,10]$. This is higher than the $0.5-3.2 \%$ frequency of scoliosis recorded in the general population [4]. Common deformity pattern is that of nonprogressive thoracic curves with T6-T8 apex; scoliosis progression has been observed in $28.6 \%$ of adolescent patients with mean curves $12^{\circ}$ and in $11.1 \%$ of adult patients despite the small curves of mean $15^{\circ}$, which is contrary to adolescent idiopathic scoliosis $[3,7]$. This may be associated with the delayed bone age observed in patients with CF [10]. There is female gender predominance in the adolescent and adult groups (Table 1). Scoliosis size or side of the curve does not correlate with the severity of lung disease, bone age, or nutritional status $[3,10]$. The presence of scoliosis is more common in patients with more severe lung disease [3].

No surgical treatment is usually required in CF patients with scoliosis due to the small curve size and low rate of progression resulting in mild deformity at skeletal maturity $[4,5,7,9,11]$. In contrast, a progressive thoracic scoliosis producing chest wall deformity as observed in our patient may further restrict lung function. A deformed chest may pose technical difficulties if lung transplant surgery was considered at a later age. In addition, our patient developed severe mechanical back pain which affected her activity level essential to preserve pulmonary function and quality of life.

A detailed preoperative assessment was critical in order to address the medical aspects of the condition and optimise the patient in view of major scoliosis surgery (Table 2). Lung function, risk of infection, bone density, and nutritional status were assessed preoperatively. The need for a multidisciplinary approach in the perioperative care of this group of patients cannot be overemphasized.

Medical comorbidities were addressed with optimisation of respiratory function through physiotherapy and prophylactic antibiotics. Bisphosphonates were introduced to improve BMD. Intraoperative blood loss was reduced using controlled hypotension and a meticulous surgical technique during exposure and instrumentation placement. Cell saver blood and products were given to accommodate anticipated blood loss. The single rod construct reduced surgical time, neurological risk, and risk of infection due to lesser implant density, achieved adequate deformity correction, and provided greater area for graft application to allow a solid fusion. At latest follow-up, our patient had an excellent cosmetic result, a balanced spine, and no respiratory complaints.

Scoliosis correction may be required in CF patients with progressive deformities which affect pulmonary function and produce pain. A meticulous preoperative assessment can reduce perioperative risks of major morbidity associated with the medical comorbidities of the condition. Posterior spinal fusion can achieve satisfactory deformity correction and a good functional outcome.

\section{Disclosure}

The authors did not receive grants or outside funding in support of their research or preparation of this paper. They did not receive payments or other benefits or a commitment or agreement to provide such benefits from a commercial entity. No commercial entity paid or directed, or agreed to pay 


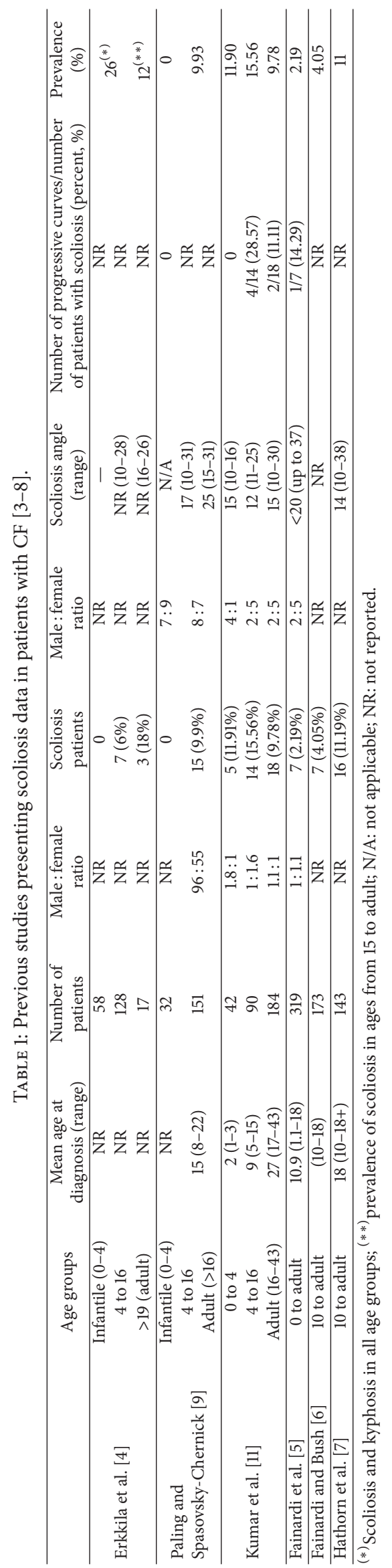


TABLE 2: Risks of scoliosis surgery in patients with CF and perioperative management.

\begin{tabular}{|c|c|c|}
\hline & Perioperative risks & Action \\
\hline \multirow{6}{*}{ Cystic fibrosis } & \multirow{2}{*}{$\begin{array}{l}\text { Poor pulmonary } \\
\text { reserve/restrictive lung disease }\end{array}$} & (1) Preoperative chest physiotherapy and increase in exercise \\
\hline & & $\begin{array}{l}\text { (2) Noninvasive ventilation, chest physiotherapy, early mobilisation } \\
\text { postoperatively }\end{array}$ \\
\hline & Recurrent infections & (1) Perioperative antibiotics \\
\hline & \multirow{2}{*}{ Poor nutrition } & (1) Emphasis on adequate oral diet \\
\hline & & (2) Nutritional supplements \\
\hline & Poor bone quality & (1) Bisphosphonate treatment \\
\hline \multirow{11}{*}{ Major surgical insult } & \multirow{6}{*}{$\begin{array}{l}\text { Increased intraoperative blood } \\
\text { loss }\end{array}$} & (1) Hypotensive anaesthesia \\
\hline & & (2) Local haemostats used \\
\hline & & (3) Meticulous, sequential spinal exposure \\
\hline & & (4) Use of less implants \\
\hline & & (5) Use of cell saver \\
\hline & & $\begin{array}{l}\text { (6) Use of allograft (no need for harvesting autologous bone from } \\
\text { other sites) }\end{array}$ \\
\hline & Reducing surgical time & (1) Use of single rod construct \\
\hline & Pain effect on & (1) Limited use of IV opioids \\
\hline & (i) chest, & (2) Aggressive chest physiotherapy \\
\hline & (ii) mobilisation, & (3) Early postoperative mobilisation \\
\hline & (iii) gastrointestinal system & (4) Supportive GI medication (including antiemetics and laxatives) \\
\hline \multirow{9}{*}{ Scoliosis correction } & Neurological & (1) Use of a single rod construct (less implant density) \\
\hline & \multirow{2}{*}{ Infection } & (1) Use of a single rod construct \\
\hline & & (2) Prophylactic antibiotics \\
\hline & Respiratory compromise & (1) Thoracoplasty not performed \\
\hline & \multirow{2}{*}{ Nonunion } & (1) Extensive bone grafting \\
\hline & & (2) Postoperative support with spinal jacket \\
\hline & \multirow{3}{*}{$\begin{array}{l}\text { Superior mesenteric artery } \\
\text { syndrome }\end{array}$} & (1) Early instigation of oral diet \\
\hline & & (2) Nutritional supplements before and after surgery \\
\hline & & (3) Early postoperative mobilisation \\
\hline
\end{tabular}

or direct, any benefits to any research fund, foundation, educational institution, or other charitable or nonprofit organisation with which the authors are affiliated or associated.

\section{Competing Interests}

The authors declare that they have no competing interests.

\section{References}

[1] Cystic Fibrosis Trust, Breathe-Life Unlimited, March 2016, https://www.cysticfibrosis.org.uk/.

[2] E. Botton, A. Saraux, H. Laselve, S. Jousse, and P. Le Goff, "Musculoskeletal manifestations in cystic fibrosis," Joint Bone Spine, vol. 70, no. 5, pp. 327-335, 2003.

[3] H. M. Buntain, R. M. Greer, P. J. Schluter et al., "Bone mineral density in Australian children, adolescents and adults with cystic fibrosis: a controlled cross sectional study," Thorax, vol. 59, no. 2, pp. 149-155, 2004.

[4] J. C. Erkkila, W. J. Warwick, and D. S. Bradford, "Spine deformities and cystic fibrosis," Clinical Orthopaedics and Related Research, vol. 131, pp. 146-150, 1978.
[5] V. Fainardi, S. D. Koo, S. P. G. Padley, S. H. Y. Lam, and A. Bush, "Prevalence of scoliosis in cystic fibrosis," Pediatric Pulmonology, vol. 48, no. 6, pp. 553-555, 2013.

[6] V. Fainardi and A. Bush, "The authors reply," Pediatric Pulmonology, vol. 49, no. 2, p. 202, 2014.

[7] C. Hathorn, A. Fall, S. McGurk, A. I. Tsirikos, and D. S. Urquhart, "Acquisition bias may have led to acceptance of the false null hypothesis that prevalence of scoliosis is the same in cystic fibrosis as the general population," Pediatric Pulmonology, vol. 49, no. 2, p. 201, 2014.

[8] M.-M. Logvinoff, G. T. Fon, L. M. Taussig, and M. J. Pitt, "Kyphosis and pulmonary function in cystic fibrosis," Clinical Pediatrics, vol. 23, no. 7, pp. 389-392, 1984.

[9] M. R. Paling and M. Spasovsky-Chernick, "Scoliosis in cystic fibrosis-an appraisal," Skeletal Radiology, vol. 8, no. 1, pp. 6366, 1982.

[10] J. Rose, J. Gamble, A. Schultz, and N. Lewiston, "Back pain and spinal deformity in cystic fibrosis," American Journal of Diseases of Children, vol. 141, no. 12, pp. 1313-1316, 1987.

[11] N. Kumar, S. Balachandran, P. A. Millner, J. M. Littlewood, S. P. Conway, and R. A. Dickson, "Scoliosis in cystic fibrosis: is it idiopathic?” Spine, vol. 29, no. 18, pp. 1990-1995, 2004. 


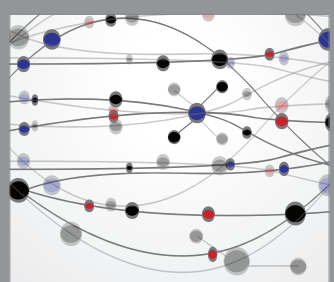

The Scientific World Journal
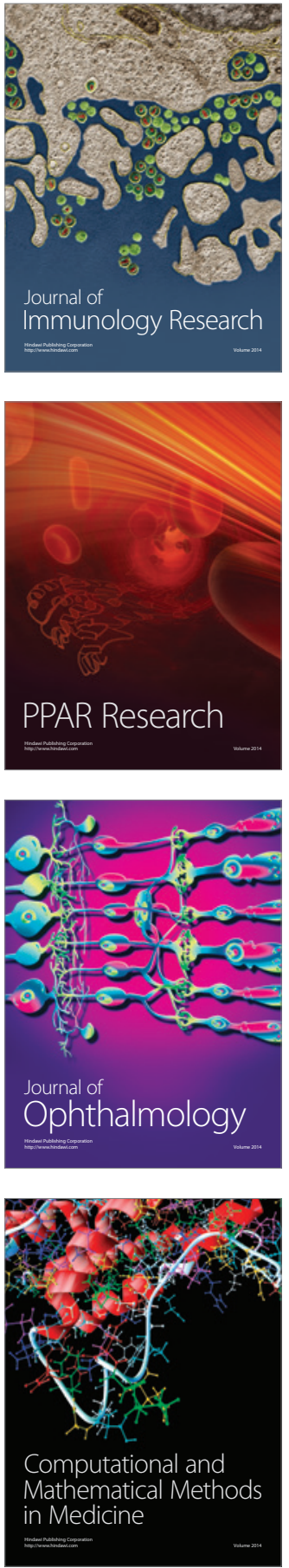

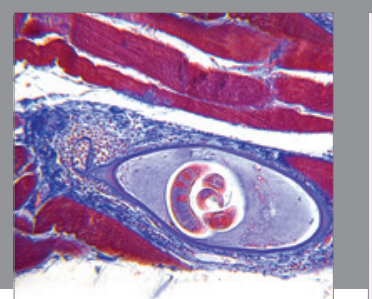

Gastroenterology Research and Practice

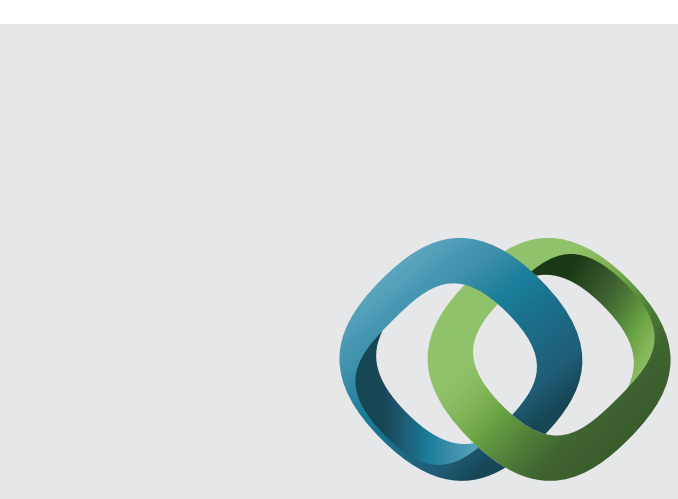

\section{Hindawi}

Submit your manuscripts at

http://www.hindawi.com
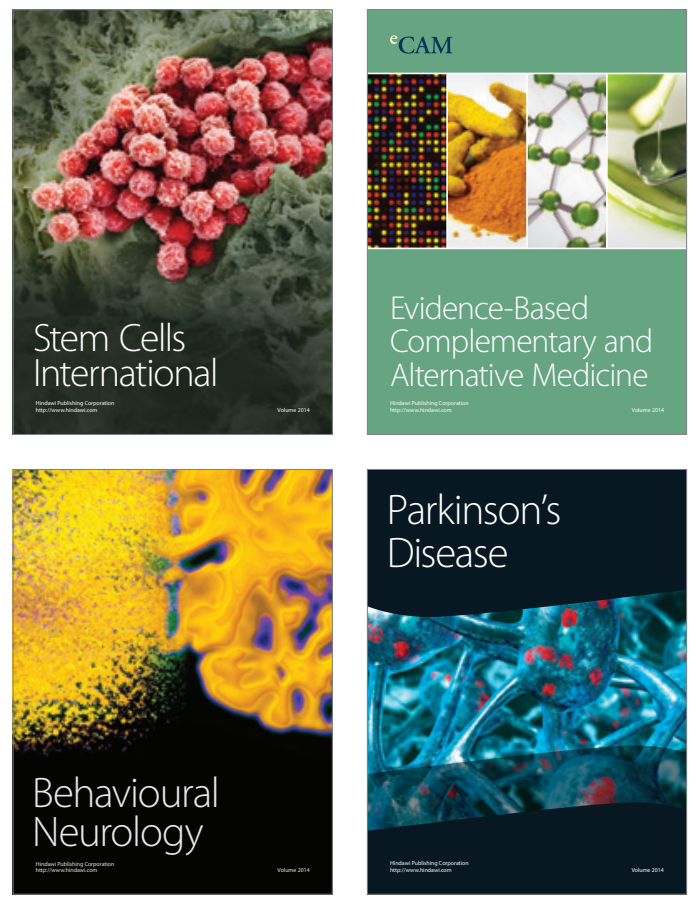
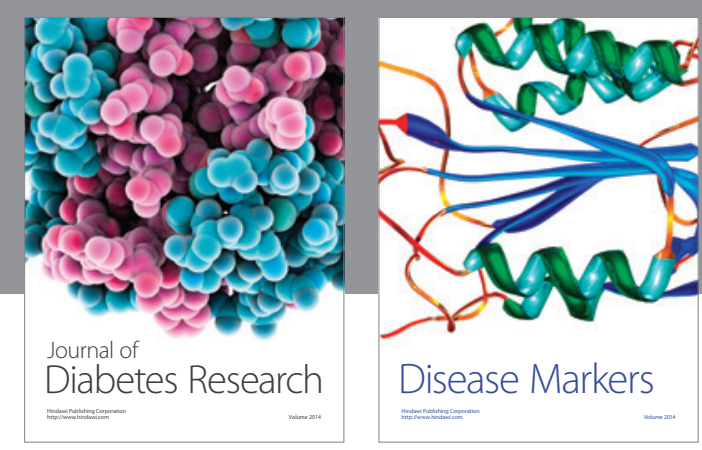

Disease Markers
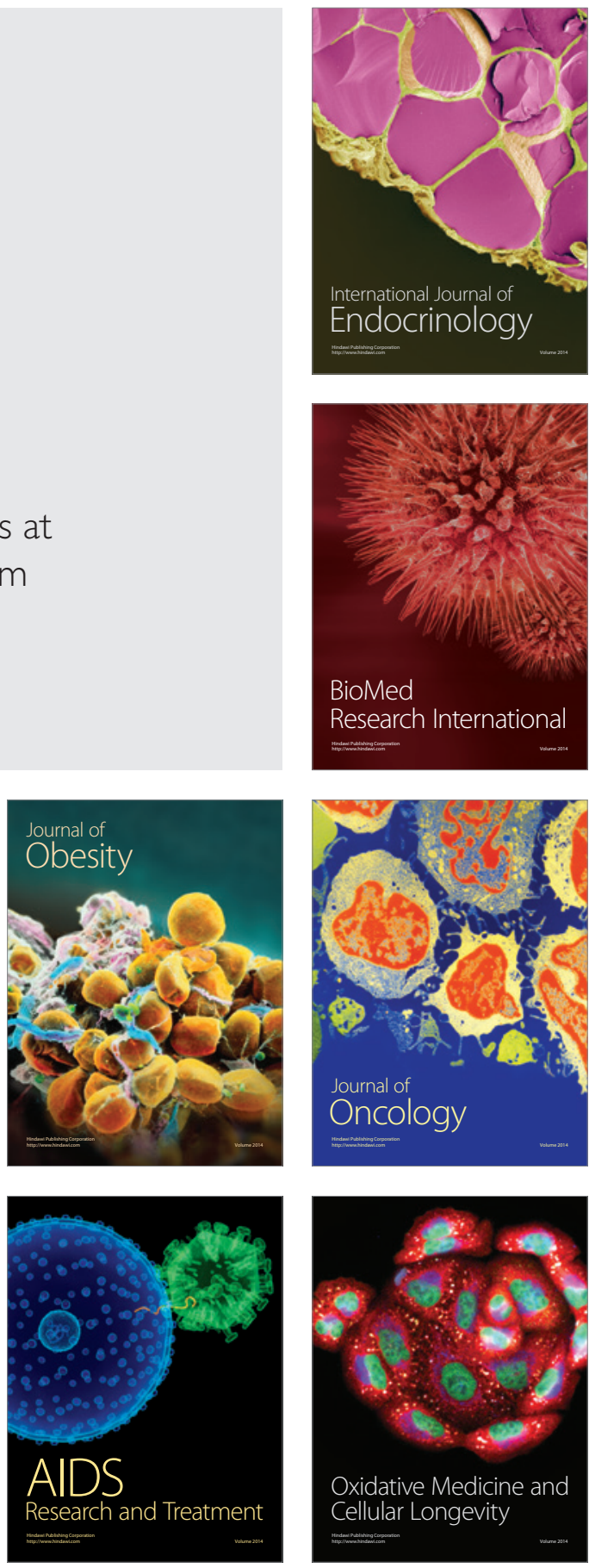